\title{
Monitoring Removal of W Layer from Ag Substrate Using Balmer- $\alpha$ Emission of Backscattered Hydrogen Atoms in Low Density Gas Discharge
}

\author{
S. Ertmer, O. Marchuk*, S. Dickheuer, S. Heuer, P.H. Mertens, \\ M. Rasinski, B. Göths, S. Brezinsek, A. Houben, \\ Ch. Linsmeier And A. Kreter
}

Forschungszentrum Jülich GmbH - Institut für Energie- und Klimaforschung-Plasmaphysik, Partner of the Trilateral Euregio Cluster (TEC), 52425 Jülich, Germany

Doi: $10.12693 /$ APhysPolA.138.643

*e-mail: o.marchuk@fz-juelich.de

\begin{abstract}
The Doppler-Shifted Reflectance Measurements diagnostic is applied for in-situ measurements of a total spectral reflectance of the sample during the transition phase induced by physical sputtering of a layer of tungsten (W) from an silver ( $\mathrm{Ag}$ ) substrate. The sputtering of a $4 \mathrm{~mm}$ layer of $\mathrm{W}$ is monitored at the Balmer- $\alpha$ line $\lambda_{0}=656 \mathrm{~nm}$ with a temporal resolution of $60 \mathrm{~s}$ corresponding to a thickness of $\approx \lambda_{0} / 100$ in the absence of other light sources. The sample was exposed to a mixed argon (Ar) and hydrogen $(\mathrm{H})$ gas discharge in the linear plasma device PSI-2, where the $\mathrm{Ar}^{+}$ions, accelerated by the applied negative potential of $-180 \mathrm{~V}$, were intrinsically used for sputtering of the deposited film. The sputtered $\mathrm{W}$ or Ag were also monitored by optical emission spectroscopy. It is shown that in low density gas discharges the Doppler-Shifted Reflectance Measurements diagnostic is, in providing the value of reflectance, extremely sensitive to the transition phase between $\mathrm{W}$ and $\mathrm{Ag}$ - as compared to resorting to the $\mathrm{W}-\mathrm{I}$ and $\mathrm{Ag}-\mathrm{I}$ emission lines intensities. It could thus be directly applied to cleaning plasma discharges with the presence of $\mathrm{Ar}$ and $\mathrm{H}$ or deuterium (D) ions.
\end{abstract}

topics: Balmer lines, sputtering, emission spectroscopy, thin films, line shape

\section{Introduction}

Optics of metallic films is a topic of extensive research of basic and applied sciences [1]. Deposition of thin films on substrates in magnetron plasmas [2] or manufacturing of optical interference coatings [3] is only one example of plasma-surface interaction studies where optical properties of films play a decisive role. However, until now, measurements of optical properties of samples require an external light source to judge on the quality of the surface or its modification during the plasma operation. Reflectance or transmission are usually obtained using coherent (lasers) and incoherent (integrating sphere) light sources. Nevertheless, there are a number of applications where the limited access to the substrate or operation in a harsh environment makes installation and application of standard techniques extremely difficult. Consequently, in future fusion devices such as ITER, for instance, the knowledge of the reflectance of the first mirror is essential $[4,5]$. A calibrating light source monitoring mirrors represent an important element of the current design of visible diagnostics as the impurity deposition leads to their degradation. It is foreseen that additional low temperature plasma discharges will be used to clean the mirror surface by physical sputtering as shown for instance in [6]. Therefore, the use of plasma emission in front of mirrors as a strong incoherent light source which replaces the measurements in the laboratory may be deemed at least attractive if not indispensable.

\section{Principles of the DSRM diagnostic}

In contrast to time-resolved measurements in the laboratory - the direct and reflected spectroscopic signals emitted by the plasma are in fact indistinguishable. The Doppler-Shifted Reflectance Measurements (DSRM) have become possible only recently [7, 8]. This new diagnostic emerged as a result of detailed study of atom-atom collisions at plasma solid interface [9]. A new approach separates the direct and reflected signal induced by backscattered $\mathrm{H}$ atoms using the Doppler effect at the kinetic energy of incident ions of $100 \mathrm{eV}$. The excitation rate of $\mathrm{H}$ atoms by collisions with Ar atoms exceeds the electron excitation rate by $2-3$ orders of magnitude at temperatures of $1-5 \mathrm{eV}$ [10]. As a result, the diagnostic operates successfully in low density plasmas with a low ionization degree. The detailed outline of the DSRM diagnostic, modelling of the spectra, including its limitation, are shown elsewhere [8, 10-12]. 
(a)

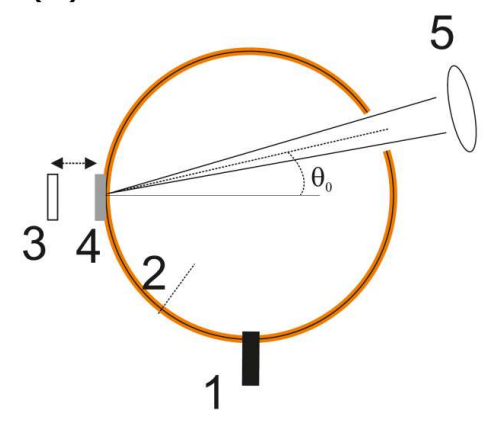

(b)

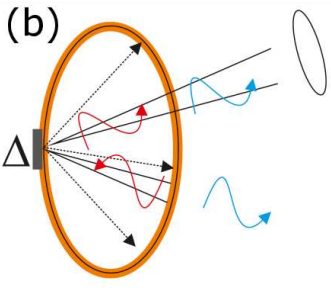

(d) (c)

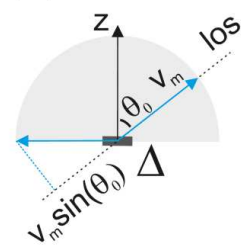

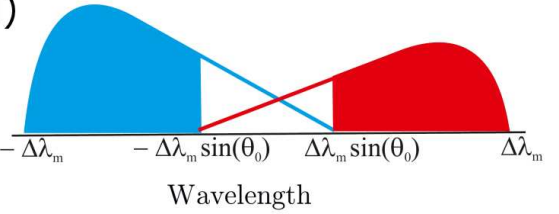

Fig. 1. Schematic setup of the measurements of total reflectance in the laboratory and using the DSRM diagnostic. (a) Measurements of the total reflectance in the laboratory: 1 is the light source, 2 is the baffle, 3 and 4 are the calibrating surface and the sample, respectively, 5 is the photo detector or the spectrometer, $\theta_{0}$ is the observation angle relative to the normal of the sample $z$. (b)-(d) Measurements of reflectance using the DSRM diagnostic. (b) shows the angular distribution of emission of backscattered atoms with the velocity $v$ at the elementary surface of the sample $\Delta$. (c) shows the energy distribution of the atoms, where $v_{m}$ is the maximal velocity of the atoms. (d) shows the schematic picture of the blue- and red-shifted signal relative to the Balmer line transition with the wavelength $\lambda_{0}$.

Figure 1 exemplifies the common and different features between the integrating sphere measurements in the laboratory and the DSRM diagnostic in the plasma. A reverse scheme of measurements of total reflectance is shown in Fig. 1a. Here, an external light source (1) illuminates an integrating sphere and multiple reflections on its surface lead finally to a diffusive illumination of the sample. Baffle (2) usually prevents the detection of direct signal from the source (1) on the sample (3). The measurements are done for the sample (3) and the calibrating surface (4) by replacing each other. By comparing both signals detected by the photo detector or spectrometer (5), which observes the sample at the angle $\theta_{0}$ relative to the sample normal, the value of total reflectance is obtained.

The emission of atoms, as shown in Fig. 1b, demonstrates common features with integrating sphere illumination at the assumption of isotropic emission and absence of strong plasma gradients along the mirror. Figure 1b shows the angular distribution of emission in front of an elementary surface $\Delta(\Delta \rightarrow 0)$ of the mirror. The situation is naturally almost the same as in the case of using the integrating sphere, except for the fact that the distribution of emission does not follow Lambert's cosine law but is defined by the distribution of atoms for this sample, i.e., it is a superposition for infinite numbers of elementary surfaces $\Delta$. The distribution of emission in the velocity space $(v, v+\mathrm{d} v)$ is homogeneous if plasma parameters do not show strong gradients along the mirror. There is no need for calibrating surface here at all as the total reflectance is determined from the Doppler effect induced by the angular and velocity distribution of the backscattered $\mathrm{H}$ atoms. So for instance, for the assumption of a perfect mirror, as shown in Fig. 1b, the detector always observes two signals: the blue-shifted one is the direct emission and the red-shifted one is the result of photon reflection on the surface.

The spectral interval and the shape of the observed emission are defined not only by the angular but also by the energy or velocity distribution of backscattered atoms, as shown in Fig. 1c. The interval of direct emission is prescribed by the projection of velocity of backscattered atoms on the line-of sight (los). It varies from $-v_{m} \sin \left(\theta_{0}\right) \rightarrow v_{m}$, where $v_{m}$ is the maximal velocity of $\mathrm{H}$ atom defined by elastic collisions of an incident ion $\mathrm{H}^{+}$with a target atom [13]. The resulting blue- and red-shifted signals are shown in Fig. 1d [10]. Until now, however, the modeling of emission is performed only in the point source approximation for unique surface element $\Delta$, i.e., no integration along the surface of the sample is performed and the temporal evolution of emission is not considered either. The undercosine distribution of the emission with $b=0.2$, where $b$ is the power of the cosine distribution of atoms, could partially be a result of the limitation of the model [12]. We apply here the DSRM diagnostic during the removal of the $\mathrm{W}$ layer from the substrate of $\mathrm{Ag}$ by physical sputtering of $\mathrm{Ar}^{+}$ions.

The aim of this paper is to investigate how sensitive is the diagnostic to the transition phase between the layer and the substrate. The previous analysis was dealing only with mono samples. Indeed, the DSRM operates in the presence of Ar gas and, strictly speaking, Ar leads by physical sputtering to the removal and degradation, depending on the material, of the optical properties of the sample at sufficiently long time of operation. For instance, the degradation of reflectance for heated $\mathrm{Al}$ target was observed within an hour of operation [11]. 
For the cooled target of $\mathrm{Al}$ and $\mathrm{Pd}$, the modification of the total reflectance was observed on the level of only a few percent after one hour of exposure [8, 11]. Therefore, it is expected that the DSRM could monitor the transition between $\mathrm{W}$ and $\mathrm{Ag}$ as the value of reflectance is determined using the ratio of the nonoverlapping part of emission in the spectrum.

\section{Experimental setup}

The experiment with the coated sample was performed in a mixed Ar- $\mathrm{H}$ gas discharge in the linear plasma device PSI-2 [14]. A schematic top view of the experimental setup is shown in Fig. 2a. The sample was installed on the side manipulator and put at the radial position corresponding to the maximum of the cylindrically symmetrical temperature and density hollow plasma profile. The electron temperature and electron density profiles, as measured by a Langmuir probe, are given in Fig. 2b. The scattering of data points results from fitting uncertainties of the analysis software for the Langmuir probe. The ions are confined by an magnetic field of about $0.085 \mathrm{~T}$ at the position of the sample [15]. The water-cooled sample was kept at room temperature during the plasma exposure. By applying a negative potential to the target of $-180 \mathrm{~V}$, the plasma ions were accelerated in the sheath. The ion impact energy was reduced by the plasma potential to $165 \mathrm{eV}$. At this energy, the sputtering is dominated by Ar ions.

For the experiments, a $4 \mu \mathrm{m} \mathrm{W}$ film was coated on top of a $13 \times 13 \mathrm{~mm}^{2}$ mirror-like polished $\mathrm{Ag}$ sample by magnetron sputtering [16]. After the coating process, a focused ion beam (FIB) - in combination with an scanning electron microscope (SEM) - was used to measure the coating thickness as shown in Fig. 3. Platinum $(\mathrm{Pt})$ is deposited on top of the $\mathrm{W}$ surface prior to the cut so that the destructions of the surface relief are avoided. The spectroscopic data reported in this paper are obtained using two instruments. The spectrometer setup was arranged like shown in Fig. 2. A high resolution spectrometer $(\mathrm{HRS})\left(\lambda / \Delta \lambda=6.6 \times 10^{5}\right)$ with an Echelle grating in the Littrow configuration was used for the observation angle $35^{\circ}$. This instrument serves to investigate the Doppler-shifted broadening in the Balmer- $\alpha$ line at $6562.79 \AA$ [17]. The spectrum was detected continuously with an integration time of $60-120 \mathrm{~s}$ to study the change in surface reflectance during the exposure. Additionally, a Princeton Instruments imaging spectrometer ACTON Series SP-750 was directed at $90^{\circ}$ to the target normal to observe the light emitted by the sputtered atoms of $\mathrm{W}-\mathrm{I}(4008.75 \AA)$ and $\mathrm{Ag}-\mathrm{I}(4055.48 \AA)$, simultaneously to the light emitted by the fast $\mathrm{H}$ atoms. This instrument also utilizes Echelle grating in the first order with a wavelength resolution of $\approx 3 \times 10^{4}$. Finally, a spectral interval of about $200 \AA$ could be observed on the chip in the current setup of the imaging spectrometer.

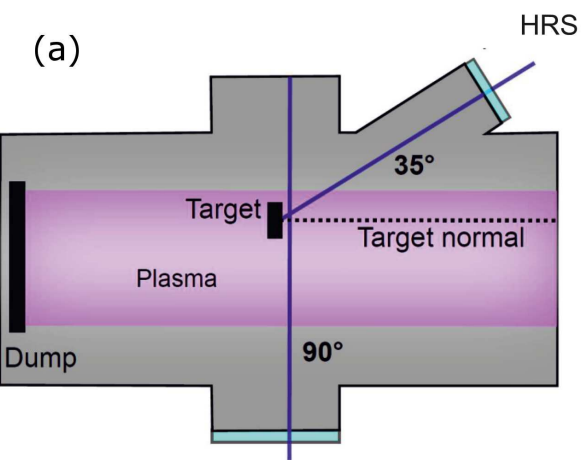

IS

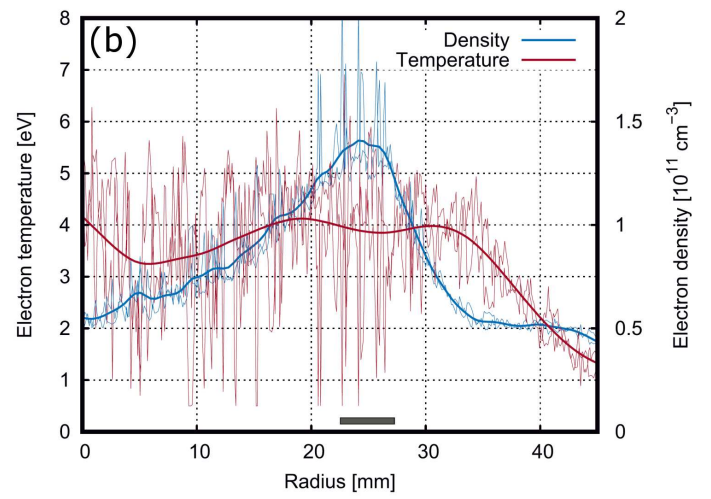

Fig. 2. (a) Schematic experimental setup at PSI-2: the sample is exposed to the plasma and the Balmer- $\alpha$ line is observed under an observation angle of $35^{\circ}$ to the surface normal using a high resolution spectrometer (HRS). The imaging spectrometer (IS) observes the light emitted by the sputtered atoms at $90^{\circ}$ to the surface normal. (b) Electron temperature and density profiles during the experiment in PSI-2 as measured by a Langmuir probe. The cathode current is $100 \mathrm{~A}$, the flows of $\mathrm{Ar}$ and $\mathrm{H} 2$ are equal to $40 \mathrm{sccm}$ each, the gas pressure in the target region equals $0.025 \mathrm{~Pa}$, the plasma potential at the radial position of the sample (grey box) measured by a Langmuir probe is $-15 \mathrm{~V}$.

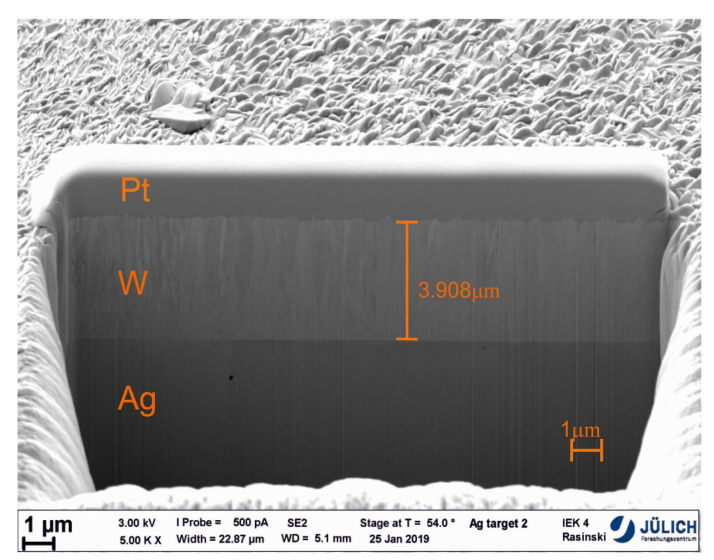

Fig. 3. The layer of $4 \mathrm{~mm} \mathrm{~W}$ on top of an Ag sample. The cut, having a width of $\approx 20 \mathrm{~mm}$ and a depth of $\approx 12 \mathrm{~mm}$, was made using a focused ion beam. The picture was taken using a scanning electron microscope. 


\section{Experimental results and analysis}

Exemplary four spectra of the $\mathrm{H}$ line observed at $35^{\circ}$ at different times during the plasma exposure are shown in Fig. 4. The full spectrum measured by high resolution instrument is presented in Fig. 4a, whereas Fig. 4b focuses only on the emission of fast atoms. Also, the passive component emitted by $\mathrm{H}$ atoms in the absence of a sample is shown using a dashed black line. As expected, no signal is detected at the blue- and red-shifted spectral intervals. By applying a negative potential to the target, the fast atoms (blue-shifted part of the spectrum) and the effect of light reflectance in the red-shifted part are detected. It can be seen that the blue-shifted component stays nearly constant over the entire exposure, whereas the red-shifted one continually increases. The strong asymmetry of the spectrum at the beginning of the discharge is replaced with symmetric profiles after $700 \mathrm{~min}$ of plasma operation.

The ratio of the red- to the blue-shifted signal measured using the DSRM diagnostic is shown in Fig. 5. The reflectance increases from 0.45 at the beginning of the discharge to the value of $0.55-0.58$ after $40 \mathrm{~min}$ of plasma operation. A possible explanation for this is a smoothing of the rough surface of $\mathrm{W}$ as seen in Fig. 3 (the zone between $\mathrm{Pt}$ and $\mathrm{W}$ ), resulting in a consequent linear increase of reflectance. The observed value of reflectance after one hour is extremely close to the most recent data on $\mathrm{W}[18]$ shown as the blue
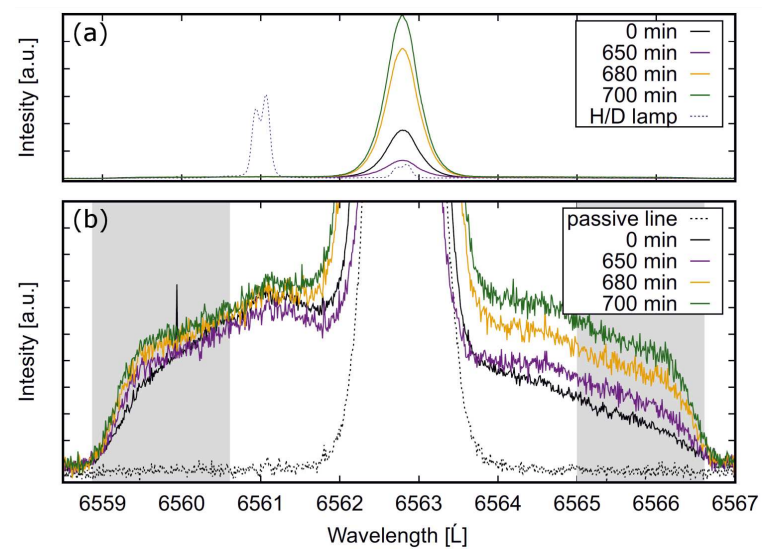

Fig. 4. Emission spectra of Balmer- $\alpha$ line induced by backscattered fast atoms in the Ar-H discharge during the transition between the $\mathrm{W}$ layer and $\mathrm{Ag}$ substrate at different time stamps. The spectra of the Balmer- $\alpha$ line, including the spectrum from the calibration lamp containing $\mathrm{H}_{2}$ and $\mathrm{D}_{2}$ gases, are shown in (a). The details of the emission induced by backscattered atoms at different times are exemplified in (b). The Balmer- $\alpha$ line observed without sample is shown using the black dashed line. The grey zones show the intervals where no overlap between blue- and red-shifted signal occur. Both spectral regions are used to obtain the value of total reflectance.

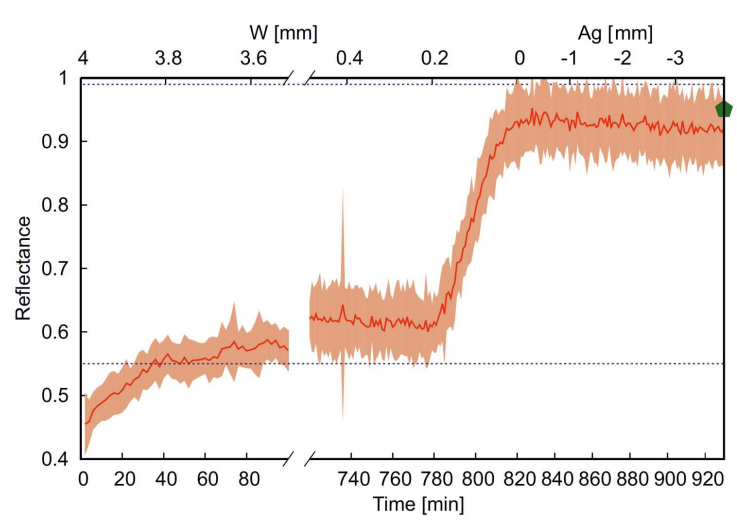

Fig. 5. Measurements of reflectance using the DSRM diagnostic. The red line shows the value of reflectance and the shadow region - the error bars. The horizontal dashed blue lines denote the values of reflectance for W $(0.549$ from [18] at $650 \mathrm{~nm}$ and $300 \mathrm{~K})$ and $\mathrm{Ag}(0.99$ from [19] at $656 \mathrm{~nm})$. The time resolution was $120 \mathrm{~s}$ for the measurements from 0 to $720 \mathrm{~min}$ and was adjusted to $60 \mathrm{~s}$ to the end of the discharge. The green diamond point shows the value of reflectance (0.95) measured using the integrating sphere in the laboratory.

dashed line in Fig. 5. After this first phase, a slow increase of reflectance in the next 8-9 h operation $(0.55 \rightarrow 0.6)$ is observed. The clear transition, i.e., the complete removal of $\mathrm{W}$ from the sample, is observed between 780 and $820 \mathrm{~min}$. The maximal detected value of reflectance is around $0.92-0.93$. There are a number of reasons why the value of $0.95-0.99[20]$ is not achieved in the plasma. The first one is the presence of oxygen below $1 \%$ in the PSI-2 plasma [21]. Also, the magnetron deposition of $\mathrm{W}$ atoms or the preferential sputtering [22] could destroy the mirror-like Ag surface so that the total reflectance reduces. After this transition phase, the reflectance remained constant with a tendency to decrease in the next $100 \mathrm{~min}$. Using the thickness of sputtered $\mathrm{W}$ on $\mathrm{Ag}$ in Fig. 3 and the time of operation of $820 \mathrm{~min}$ required to observe the transition, the density of $\mathrm{Ar}^{+}$species in the plasma could be calculated. Indeed, taking into account the expression for the sputtering rate [23]:

$$
R=Y \frac{J_{i}}{n_{A} e}
$$

the current density $J_{i}$ and the ion density of $\mathrm{Ar}^{+}$ incident on the sample could be estimated. Here, $R$ is the sputtering rate $(\mathrm{cm} / \mathrm{s}), Y$ is the sputtering yield, $J_{i}=n_{i} e v_{i}$ is the current density to the sample $\left(\mathrm{C} /\left(\mathrm{cm}^{2} \mathrm{~s}\right)\right), n_{A}$ is the density of the sputtered atoms of $\mathrm{W}\left(\mathrm{cm}^{-3}\right), v_{i}$ is the velocity of the $\mathrm{Ar}^{+}$ions at the sample $(\mathrm{cm} / \mathrm{s})$ and $e$ is the elementary charge $(\mathrm{C})$. We are aware that also the $\mathrm{ArH}^{+}$could be present in such plasmas, however we do not expect significant difference in the sputtering process caused by $\mathrm{Ar}^{+}$ 
or $\mathrm{ArH}^{+}$ions. Taking the kinetic energy of the $\mathrm{Ar}^{+}$ions $E_{i}=165 \mathrm{eV}$, the density of W atoms of $n_{A}=1.9 \mathrm{~g} / \mathrm{cm}^{3}$, the sputtering yield $Y=0.15$ at $165 \mathrm{eV}$ from $[24]$ and $e=1.602 \times 10^{-19} \mathrm{C}$ we obtain the density of Ar ions of $n_{\mathrm{Ar}+}^{t}=1.4 \times 10^{9} \mathrm{~cm}^{-3}$ at the target. It corresponds to the current density of $\mathrm{Ar}^{+}$of $0.537 \mathrm{~mA} / \mathrm{cm}^{2}$.

To obtain the electron density in mixed plasmas, the mass $\mu$ of the average ion has to be used. The value of $\mu$ is largely unknown in our experiment as it depends on relative concentration of $\mathrm{Ar}$ and $\mathrm{H}$ ions. Until now we used the value $\mu \approx 20$ corresponding to the relative flux of $\mathrm{H}_{2}$ and Ar atoms. Here, the density of $\mathrm{Ar}$ ions at the probe position (upstream) was estimated using the expression $n_{i}^{u} \approx 2 n_{i}^{t} \sqrt{E_{i} / T_{e}}$, where $T_{e}=4 \mathrm{eV}$ is the electron temperature as measured by the probe and $n_{i}^{t}$ is the ion density at the target. We further iterated the average mass $\mu$ till the agreement between the ion density measured using sputtering rate and using the Langmuir probe experimental data was achieved. In the calculation of electron density profiles the mass $\mu=6.1$ was further used.

In fact, the extrapolation of the results of [25] (see Fig. 4) to the gas flow values used here provides a qualitative agreement with our data: the flux of $\mathrm{Ar}^{+}$is reduced considerably compared to the flux of $\mathrm{H}^{+}$. The temporal resolution of $60 \mathrm{~s}$ results in a removal rate of material of approximately $5 \mathrm{~nm}$. Thus, during the measurements of one spectrum the plasma removes the thickness $l \approx \lambda / 100$ of $\mathrm{W}$, where $\lambda$ is the wavelength in the visible range. Taking into account that the surface with the irregularities $\sigma \approx \lambda / 20$ is considered as an ideal mirror, we demonstrated here for the first time that the DSRM diagnostic is ideally suited to characterize optical properties of the surfaces as $l<<\sigma$ on the macroscopic scale. Below, we compare the measured time of transition between $\mathrm{W}$ and $\mathrm{Ag}$ and expectations. Indeed, e-folding length of energy density in the metal equals to [1]:

$$
d=\frac{\lambda}{4 \pi \kappa},
$$

where $\kappa$ is the extinction coefficient for the material $(\kappa \approx 3$ for $\mathrm{W})$. Thus, in the case of a smooth interface between $\mathrm{W}$ and $\mathrm{Ag}$ one has to detect the transition between two values of reflectance within of 5-6 spectra. Here, instead, one observes the transition within 40 spectra, corresponding to a thickness $d$ of $200 \mathrm{~nm}$. Thus, the transition time measured by DSRM shows that the Ag surface become diffusive most probably either during the magnetron sputtering or during the removal of W from Ag substrate and not afterwards. The post measurements of reflectance in the laboratory also demonstrated a purely diffusive reflectance of the Ag sample with the value of reflectance of $0.95 \pm 0.03$. We are going to investigate this effect in the near future by deposition layers of different materials at other experimental conditions in magnetron or using other techniques such as evaporation.

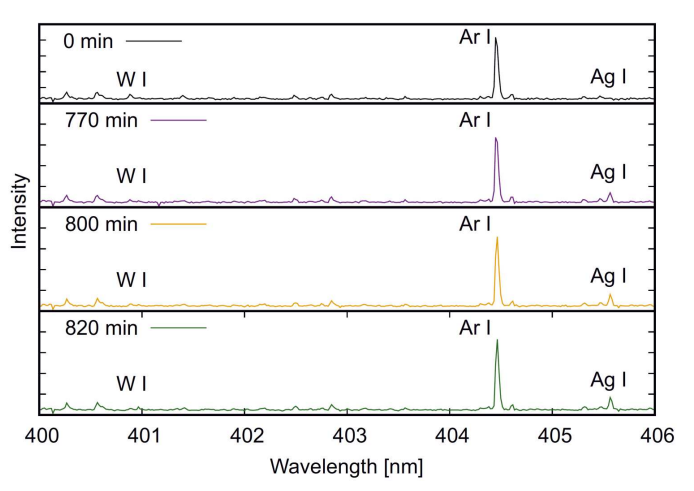

Fig. 6. Emission spectra of sputtered $\mathrm{W}-\mathrm{I}$ and $\mathrm{Ag}-\mathrm{I}$ lines measured using the imaging spectrometer (IS) at the line-of-sight parallel to the surface.

In addition to DSRM measurements, the monitoring of sputtering was performed using the emission spectroscopy of $\mathrm{W}-\mathrm{I}$ and Ag-I lines. The spectra interval around one of the strongest lines of $\mathrm{W}-\mathrm{I}$, observed for instance in fusion plasma [26], was selected for the analysis, as no switching between spectral ranges was required. Even though the integration time of the imaging spectrometer was $5 \mathrm{~min}$, by the factor of five longer compared to the high resolution spectrometer, the detected signal of the sputtered particles was close to the detection limit. Spectra detected with this imaging spectrometer are shown in Fig. 6. For the eroded material, the neutral tungsten transition (W-I) at $4008.75 \AA$ [17] and the neutral silver transition $(\mathrm{Ag}-\mathrm{I})$ at $4055.47 \AA$ [17] are marked in the spectrum. Furthermore, the neutral argon transition (Ar-I) at 4044.42 $\AA$ [17] is labelled. Under plasma conditions used, the sputtered particles are excited by electron impact excitation, whereas the fast $\mathrm{H}$ atoms get excited by atom-atom collisions. It can be seen how the light emitted by tungsten, and thereby the sputtering of tungsten, is decreasing and the $\mathrm{Ag}$ signal is increasing over time. The increase of $\mathrm{Ag}$ is obviously stronger as the decrease of $\mathrm{W}$ due to a different sputtering yield. Consequently, for instance for $\mathrm{Ag}$ it equals 1.11, whereas for $\mathrm{W}-\mathrm{I}$ it is 0.15 only. The $\mathrm{W}$ signal is strongly reduced already after $770 \mathrm{~min}$ in comparison to the first spectra and the Ag signal is already increased, even though DSRM still does not show a strong gradient in transition. It is interesting that the detected value of reflectance of 0.6 at time stamp of 770 min exceeds the value of reflectance for $\mathrm{W}$. The emission spectrum confirms it as the Ag atoms already appear on the surface. The DSRM diagnostic determines the reflectance at a point of $\approx 3 \mathrm{~mm}$ diameter. Due to the gradient in the plasma parameter, local tungsten islands can survive contributing to the W-I lines emission, whereas in the center of the sample only the substrate is observed.

The comparison demonstrates the major advantage of DSRM approach, namely the excitation of lines by atom-atom impact is much more efficient 
in the low temperature gas discharges with the ionization degree on the order of $1-3 \%$. The sputtering of $\mathrm{W}-\mathrm{I}$ is extremely weak and the signal scales as $n_{e}^{2}$, where $n_{e}$ is the electron density. In the case of atom-atom excitation the signal is 100-1000 times larger. Also, a weak variation of electron temperature leads to strong variation of the line intensities. In the case of DSRM approach this is not the case. The reduction of applied negative surface potential to values of $-100 \mathrm{~V}$ being the optimal for the operation of the DSRM diagnostic, leads to a further decrease of the measured signal.

\section{Conclusion}

The DSRM diagnostic was applied as an in-situ monitor of the transition phase between metallic surfaces caused by sputtering. Obviously in low pressure gas discharges, the line shapes of backscattered atoms monitor the transition between two metallic surfaces much more effectively compared to the classical emission spectroscopy induced by electron impact. The analysis is based only on the value of directly measured total reflectance and does not require an additional modelling.

In this paper, we measured the transition phase between a deposited W layer and the Ag substrate. We operated the DSRM diagnostic with a time resolution of $60-120 \mathrm{~s}$ which resulted in sputtered thickness of $\lambda / 100$ for $W$. The rapid growth of reflectance caused by the transition phase between $\mathrm{W}$ and $\mathrm{Ag}$ was observed on depths of $200 \mathrm{~nm}$ during $40 \mathrm{~min}$ of plasma exposure. This value exceeds the expected decay length of energy density in $\mathrm{W}$, indicating that the interface between $\mathrm{W}$ and $\mathrm{Ag}$ is not smooth. It leads to a considerable diffusive component of the Ag substrate. The post analysis confirmed the DSRM data: a pure diffusive component of reflectance for $\mathrm{Ag}$ was detected using the integrating sphere measurements (green diamond point in Fig. 5). The value of total reflectance of 0.92 was found in excellent agreement with the value of $0.95 \pm 0.005$ in the laboratory. In this experiment, only the total value of reflectance was monitored and no attempts were undertaken to distinguish between the specular and diffusive part using polarization by light reflection $\left(\theta_{0} \approx 50^{\circ}-70^{\circ}\right)$ [12]. It seems that this novel technique is rather effective in investigating damages on the interface between materials, caused, for instance, by different regimes of films production. Another field of application is the cleaning processes. We are going to address both topics in the near future.

\section{Acknowledgments}

The authors thank V. Kotov and G. Sergienko for helpful discussion on Langmuir probe measurements in mixed plasmas. This work has been carried out within the framework of the EUROfusion Consortium and has received funding from the Euratom Research and Training Programme 2014-2018 and 2019-2020 under grant agreement No 633053. The views and opinions expressed herein do not necessarily reflect those of the European Commission.

\section{References}

[1] M. Born, E. Wolf, Principles of Optics, 7th ed., Cambridge University Press, 2002 p. 735 .

[2] D. Rohde, H. Kersten, C. Eggs, R. Hippler, Thin Solid Films 305, 164 (1997).

[3] K. Juškevičius, R. Buzelis, G. Abromavičius, R. Samuilovas, S. Abbas, A. Belosludtsev, R. Drazdys, and S. Kičas Opt. Mater. Express 7, 3598 (2017).

[4] W. Biel, T. Baross, P. Bourauel et al., Fusion Eng. and Design 86, 548 (2011).

[5] Ph. Mertens, J. Fusion Energy 38, 1 (2019).

[6] L. Moser, L. Marot, R. Steiner et al., Phys. Scr. T170, 014047 (2017).

[7] O. Marchuk, C. Brandt, A. Pospieszczyk, Verfahren zur Bestimmung der Oberflächeneigenschaften von Targets (Method for Determining the Surface Characteristics of Targets) Patent DE102016002270B3, 2017; US10444156B2, 2019.

[8] S. Dickheuer, O. Marchuk, C. Brandt et al., Rev. Sci. Instrum. 89, 063112 (2018).

[9] O. Marchuk, C. Brandt, A. Pospieszczyk, M. Reinhart, S. Brezinsek, B. Unterberg, S. Dickheuer J. Phys. B: At. Mol. Opt. Phys. 51, 025702 (2017).

[10] O. Marchuk, S. Dickheuer, S. Ertmer et al., Atoms 7(3), 81 (2019).

[11] S. Dickheuer, O. Marchuk, S. Ertmer, A. Goriaev, M. Ialovega, B. Göths, Y. Krasikov, Ph. Mertens, A. Kreter, Nucl. Mater. Energy 17, 302 (2018).

[12] S. Dickheuer, O. Marchuk, Y. Krasikov et al., Phys. Plasma 26, 073513 (2019).

[13] D.P. Smith, Surf. Science 25, 171 (1971).

[14] A. Kreter, C. Brandt, A. Huber, S. Kraus, S. Möller, M. Reinhart, B. Schweer, G. Sergienko, B. Unterberg, Fusion Sci. Technology 68, 8 (2015).

[15] S. Dickheuer, O. Marchuk, T.V. Tsankov, D. Luggenhölscher, U. Czarnetzki, W. Gromelski, S. Ertmer, A. Kreter, Atoms 7, 48 (2019).

[16] S. Heuer, Ph.D. thesis, Charakterisierung gradierter Eisen/Wolfram-Schichten für die erste Wand von Fusionsreaktoren 2017, p. 32 . 
[17] A. Kramida, Yu. Ralchenko, J. Reader et al., NIST Atomic Spectra Database (ver. 5.6.1), 2018.

[18] M. Minissale, C. Pardanaud, R. Bisson, L. Gallais, J. Phys. D 50, 455601 (2017).

[19] P.B. Johnson, R.W. Christy, Phys. Rev. B 6, 4370 (1972).

[20] M.N. Polyanskiy, Refractive index database.

[21] J. Schmitz, A. Mutzke, A. Litnovsky et al., J. Nucl. Mater. 526, 1517672 (2019).

[22] S. Berg, I.V. Katardjiev, J. Vac. Sci. Technol. A 17, 1916 (1999).

[23] M.A. Lieberman, A.J. Lichtenberg, Plasma Discharges and Materials Processing, 2nd ed., J. Wiley \& Sons, Inc., Hoboken (NJ) 2005, p. 564.
[24] W. Eckstein, "Calculated Sputtering, Reflection and Range Values", IPP Report 9/132, 2002.

[25] M. Reinhart, Ph.D. thesis, Influence of Impurities on the Fuel Retention in Fusion Reactors, 2015 p. 57.

[26] S. Brezinsek, M. Laengner, J.W. Coenen, M.G. O'Mullane, A. Pospieszczyk, G. Sergienko, U. Samm, Phys. Scr. T170, 014052 (2017). 